\title{
Effects of bionic mechanical stimulation on the properties of engineered cartilage tissue
}

Zhiyan Hao ${ }^{1,2}$, Sen Wang ${ }^{1,2}$, Jichang $\mathrm{Nie}^{1,2}$, Dichen $\mathrm{Li}^{1,2}$, Ao Fang ${ }^{1,2}$, Jianfeng Kang ${ }^{3}$, Chaozong Liu ${ }^{4}$,

Wang $\operatorname{Ling}^{1,2, *}$

${ }^{1}$ State Key Laboratory for Manufacturing System Engineering, Xi'an Jiaotong University, 710054, Xi'an, Shaanxi, China

${ }^{2}$ School of Mechanical Engineering, Xi'an Jiaotong University, 710054, Xi'an, ShaanXi, China

${ }^{3}$ Jihua Laboratory, 528200, Foshan, Guangdong, China

${ }^{4}$ John Scale Centre for Biomedical Engineering, University College London, Royal National Orthopaedic

Hospital, Stanmore, HA7 4LP, UK

*Corresponding authors

Ling Wang

E-mail: menlwang@mail.xjtu.edu.cn (Ling Wang),

Tel.: +862983395382

Address: State Key Laboratory for Manufacturing Systems Engineering

Xi’an Jiaotong University, Xi'an 710054, Shaanxi, China

\begin{abstract}
Tissue-engineered cartilage (TEC) remains a potential alternative for the repair of articular cartilage defects. However, there has been a significant different between the properties of TEC and those of natural cartilage. Studies have shown that mechanical stimulation such as compressive load, can help regulate matrix secretion in TEC, thus affecting its biomechanical properties. However, the influences of shear induced from the tissue fluid phase have not been well studied, and may play an important role in tissue regeneration especially when integrated with the compressive load. Therefore, the aim of this study
\end{abstract}


was to quantitatively investigate the effects of combined loading mechanisms on TEC in-vitro. A bespoke biosimulator was built to incorporate the coupled motion of compression, friction and shear. The specimens, encapsulating freshly isolated rabbit chondrocytes in a hydrogel, were cultured within the biosimulator under various mechanical stimulations for four weeks, and the tissue activity, matrix contents and the mechanical properties were examined. Study groups were categorized according to different mechanical stimulation combinations, including strain (5\% 20\% at 5\% intervals) and frequency $(0.25 \mathrm{~Hz}, 0.5 \mathrm{~Hz}, 1 \mathrm{~Hz})$, and the effects on tissue behaviour were investigated. The results indicated that a larger strain and higher frequency were more favourable for the specimen in terms of the cell proliferation and extracellular matrix synthesis. Moreover, the combined mechanical stimulation was more beneficial to matrix secretion than the single loading motion. However, the contribution of the combined mechanical stimulation to the engineered cartilaginous tissue matrix was not sufficient to impede biodegradation of the tissue with culture time.

Key words: Bionic mechanical stimulation; Tissue-engineered cartilage; Biosimulator; Shear

\section{Introduction}

Bone and rheumatoid arthritis are common diseases that affect the articular cartilage tissue of adults due to arthritis, trauma, or tumors. Because articular cartilage has no blood vessels, lymphatic vessels, or nerves, chondrocytes have poor proliferation rate and a low regeneration capacity, thus unable to migrate to damaged sites, which makes it difficult to repair defects on its own[1,2]. Clinical repair methods include cartilage transplantation[3], subchondral bone drilling and microfracture. Cartilage transplantation involves preparation and preservation of a graft, coupled with maintenance of the cells during the operation[3]. Although subchondral bone drilling and microfracture[4] can achieve a shortterm treatment effect, the regenerated cartilage is fibrocartilage which has an inferior property than 
hyaline cartilage and can not hold long in the joint, which might result in further joint degeneration in the long run.

Tissue engineering has provided a new treatment method for osteochondral repair[5]. Generally, an engineered tissue is composed of a scaffold, cells, and necessary growth factors[6,7], which can effectively repair cartilage damage and restore cartilage function[8]. At present, although tissue engineering can produce hyaline cartilage similar in appearance/morphology to articular cartilage, its properties, such as compression modulus and permeability coefficient, are still far from those of natural cartilage[9-11]. Normal articular cartilage is always subjected to complex mechanical loads during daily activities[12]. The poor environment of the joint site after scaffold implantation can easily lead to hardening and cracking of the host, the tissue itself degenerates due to the poor environment and nutrient deficiency[13]. Therefore, researchers have attempted to improve the performance of tissue-engineered cartilage via external action. Studies have found that proper stimulation can affect the cartilaginous tissue morphology and gene expression; regulate the synthesis and secretion of matrix components such as GAGs and collagen[14-17]; and play an important role in maintaining the normal structure and function of articular cartilage. To date, most studies of the effects of mechanical stimuli on tissue cartilage have focused on a single type, such as fluid shear stress, liquid pressure, stretching, and simple compression[18-23]. Omata et al. demonstrated that cyclic compressive loading can benefit tissue engineering chondrocyte matrix secretion and its mechanical properties[19]. Tony et al. applied fluid shear to an agarose scaffold compounded with porcine articular chondrocytes and found that the fluid shear force helped promote secretion of tissue-engineered cartilage matrix and improved the mechanical properties[22]. In general, articular motion is the combination of compression load and shear stress[24]. Therefore, it is of great significance to study the effects of composite forms of mechanical stimulation 
on tissue-engineered cartilage, to find an optimized stimulus to regulate and improve the structure and mechanics of tissue during in vitro culture[25].

In this paper, tissue-engineered cartilage samples were constructed through encapsulation of freshly derived chondrocytes, and various loading schemes were applied to the engineered cartilaginous tissue via a bespoke biosimulator to study the effects of combined mechanical stimulation on tissue morphology and properties during long-term in vitro culture[26].

\section{Equipment and Materials}

\subsection{Bespoke biosimulator}

A biosimulator was designed and built in-house for functional stimulation of engineered cartilaginous tissue and is shown in Fig. 1. The simulator includes three parts: a mechanical stimulation actuator, a controller part and a sterile environment. The actuator is used for clamping the sample as well as directly applying stimulation to the sample; the controller is used for inputting the motion and loading parameters to control the movement of the actuator; the sterile environment ensures the absence of contamination throughout the dynamic culture process. The mechanical stimulation actuator is mainly composed of horizontal motion unit and vertical loading unit. The vertical loading unit can apply dynamic compressive load to the tissue samples by reciprocating the indenter vertically at a specific frequency and amplitude relative to the experimental samples. The lower platform holding four identical stations of PVA fixtures for the tissue samples can be driven to reciprocate horizontally, which can generate shear to the interfacing surface of the tissue samples and the indenter tissue. The vertical stroke was $35 \mathrm{~mm}$, the horizontal stroke was $28 \mathrm{~mm}$, the maximum speed of vertical and horizontal motion was $20 \mathrm{~mm} / \mathrm{s}$, and the vertical loading frequency was $2 \mathrm{~Hz}$. 


\subsection{Cell isolation and Encapsulation}

\subsubsection{Cell isolation}

Chondrocytes derived from the limbs of one-day-old New Zealand white rabbits were selected in this study. The rabbits were sacrificed by cervical dislocation, and the cartilage was dissected from the elbow joints of the forelegs and knee joints of the hind limbs which were disinfected with $75 \%$ alcohol. Samples were placed in a petri dish with PBS and cut into pieces. After being washed 3 times with PBS, the specimens were digested at $37^{\circ} \mathrm{C}$, in DMEM solution containing $10 \mathrm{mM}$ Hepes- $\mathrm{NaOH}, \mathrm{pH} 7.4,2 \%$ fetal calf serum (Seromed, Berlin) $2 \mathrm{mM}$ glutamine, $500 \mathrm{U} \mathrm{ml}^{-1}$ penicillin, $500 \mathrm{U} \mathrm{ml}^{-1}$ streptomycin and 125 $\mathrm{U} \mathrm{ml}^{-1}$ collagenase (Sigma C6885) for $4 \mathrm{~h}$ under constant oscillation. The supernatant in the centrifuge tube was removed at $4 \mathrm{~h}$ intervals, and the suspension was filtered off with a cell sieve (FALCON, 352340). The supernatant was transferred into a $15 \mathrm{ml}$ centrifuge tube, centrifuged at $1300 \mathrm{rmp}$ for 5 mins, and then precipitated and prepared for encapsulation[27,28].

\subsubsection{Cell encapsulation}

The preparation process employed a casting methodology and is shown in Fig. 2. Sodium alginate solution $(2 \%(\mathrm{~m} / \mathrm{v})$, sodium alginate from brown algea, st2116-100g, Reagent Grade, Life Science Products \& Services) was used to prepare the sodium alginate cell suspension with a chondrocyte density of $3 \times 10^{6} \mathrm{cell} / \mathrm{s} / \mathrm{ml}$. Chondrocytes and sodium alginate solution were added to the $50 \mathrm{ml}$ centrifuge tube according to the cell density $\left(3 \times 10^{6} \mathrm{cell} / \mathrm{s} / \mathrm{ml}\right)$, and the centrifuge tube was placed on a vortex mixer to oscillate at high speed to homogenize the suspension. The mould was removed from the disinfectant, washed with normal saline 3 times and placed in an ultraclean table for air drying. Afterwards, the sodium alginate cell suspension was injected into the mould along the sidewall of the mould cavity. A sterile membrane was laid on the top surface of the mould to eliminate air bubbles. Next, a pressure plate was 
placed on the filter membrane and pressed on the mould parting surface. $\mathrm{CaCl}_{2}$ solution (Tianjin Hengxing Chemical Reagent Manufacturing Co., Ltd.) was injected into the mould through the liquid filling hole on the pressure plate. The volume ratio of the calcium alginate cell suspension to the $\mathrm{CaCl} 2$ solution was 1: 1 . The mould was transferred into the cell incubator and incubated for $4 \mathrm{~h}$. Then, the press plate was removed, the $\mathrm{CaCl}_{2}$ solution was suctioned out, the filter membrane was gently opened, and tissue-engineered cartilage of $9 \mathrm{~mm} \times 4 \mathrm{~mm} \times 5 \mathrm{~mm}$ was obtained by stripping.

\subsection{Bionic mechanical stimulation}

In human daily activities, the mechanical stimulation of articular cartilage primarily involves pressure, friction, and shear. The main forms of articular cartilage stress vary under different motion states. To identify the roles played by the compressive load, frictional velocity and loading frequency, seven combined loading strategies were planned, as shown in Table 1.

Among them, in group $\mathrm{P}$, a simple compressive load was applied during the dynamic culture process to simulate the pressure of the articular cartilage during standing. During the dynamic culture process, a sliding compressive load was applied in the S, SA2, SA1, SF2, and SF1 groups to simulate the combined effects of compression, friction, and shear on articular cartilage during human movement, and a controlled variable method was adopted in which the compression amplitude changes in S, SA2, and SA1 were $20 \%, 10 \%$, and 5\%, respectively. The frequency changes in the S, SF2, and SF1 groups were $1 \mathrm{~Hz}, 0.5 \mathrm{~Hz}$ and $0.25 \mathrm{~Hz}$. The experimental groups were dynamically cultured in the biosimulator for $1.5 \mathrm{~h}$ per day. In addition to the exercise time, the samples were placed in a flat culture flask in a $\mathrm{CO}_{2}$ incubator for static culture. For comparison, the control group $\mathrm{C}$ was only subjected to static culture in the incubator throughout the experiment. The samples were cultured for up to 4 weeks and were examined at the midpoint. 


\subsection{Tissue Examination Methodologies}

\subsubsection{Tissue Activity Test}

The freezing slice method was used to obtain $200 \mu \mathrm{m}$ thickness slices. A LIVE/DEAD Viability/Cytotoxicity Kit (L3224, Thermo, USA) was utilized to study the cell viability of the engineered cartilaginous tissue. The living cells were stained with a green fluorescent marker, and the dead cells were stained with a red marker. The engineered cartilaginous tissue was collected, fixed in $10 \%$ neutral buffered formalin, processed using standard histological techniques and stained with haematoxylin and eosin (H\&E). Laser scanning confocal microscopy (LSCM) (A1, Nikon, Japan) was used to image the stained cells.

\subsubsection{Cell Proliferation}

Cell proliferation was determined with Cell Counting Kit-8 (CCK-8) according to the manufacturer's protocol (Dongren Chemical Technology Shanghai Co., Ltd). CCK-8 allows convenient assays using WST-8 reagent, which is bio-reduced by cellular dehydrogenases to an orange formazan product. The amount of formazan produced is directly proportional to the number of living cells.[29] CCK-8 solutions were added to each sample and the incubation was continued for an additional $4 \mathrm{~h}$. The experiment was performed with five wells per group. The absorbance of each well was measured using a microplate reader $(1510$, Thermo, USA).

\subsubsection{Cell Matrix Secretion}

The secretion of extracellular matrix in tissue-engineered cartilage was evaluated by detecting the collagen and GAG content in the matrix. The samples were dissolved in sodium citrate solution (55 $\mathrm{mM}$ / L sodium citrate (analytical grade, Tianjin Tianli Chemical Reagent Co., Ltd.) + 0.15 M/L sodium chloride solution (analytical grade, Hongyan Reagent Factory, Tianjin Hedong District)) with ultrapure 
water at $4{ }^{\circ} \mathrm{C}$. The supernatant obtained by centrifugation was a solution of GAGs and hydroxyproline in the further matrix. The sediment was dissolved after centrifugation in papain solution $(50 \mu \mathrm{g} / \mathrm{ml}$ papain (P0150, Xi'an Guoan Biotechnology Co., Ltd.)) prepared with PBS + 5m M/L EDTA (analytical grade, Tianjin Shengao Chemical Reagent Co., Ltd.) + 5 mM/L L-cysteine (C108237-100 g, Aladdin) with PBS at $60{ }^{\circ} \mathrm{C}$. Then, the supernatant obtained by centrifugation was a solution of cell-associated matrix GAGs and hydroxyproline in the soluble matrix. Enzyme linked immunosorbent assay (ELISA) was used to detect the concentration of hydroxyproline and GAGs in the sample solution. Hydroxyproline occupies a specific proportion in collagen. Quantitative determination of hydroxyproline content is a common method for quantitative detection of collagen.

\subsubsection{Evaluation of Mechanical Properties}

Samples at 0, 2 and 4 weeks culturing points were taken from each group and subjected to an unconfined compression test using a testing machine. (ETM103A, Shenzhen Wantest Testing Equipment Co., Ltd.). The compression stroke was set to be within $1.5 \mathrm{~mm}$ vertically, and the compression speed was set to be $3 \mathrm{~mm} / \mathrm{min}$. The compressive modulus can be calculated via curve fitting of the stress-strain curve.

\section{Results}

The Live/Dead staining results for samples at 0, 2 and 4 week culturing times are shown in Fig. 3. The chondrocytes were found to be evenly distributed in the samples of all groups, and the cells were in good condition at the beginning of the culture. After two and four weeks of culture, dead cells appeared for all groups, but could be neglectable compared to the large number of viable cells. The results also indicate that the chondrocytes always maintained a spherical shape in the calcium alginate scaffold, and did not show significant differences in terms of the morphology or distribution among different groups. Sections of samples at $10 \mu \mathrm{m}$ thickness were prepared along the vertical plane in align with the rubbing 
direction after 4 weeks of culture, whose histological studying results are shown in Fig. 4[30]. Each group of cells in the scaffolds grown well and the form of cell was normal. The cells in the sliding compression group (group S) and the single compression group (group P) showed some aggregation in the pores of the scaffolds, whilst the cells in the control group were relatively dispersed.

The cell proliferation for samples taken on the 2 and 4 weeks culturing time were examined and presented in Fig. 5. After two weeks of dynamic cultivation, the cell proliferation of all groups were increased significantly, and the proliferation rate of all dynamic culturing groups were higher than that of the static culturing control group (group C). After 4 weeks of culture, the cell proliferation rate of all groups were still significantly higher than that at the starting point, but the proliferation rate decreased compared with those of the second week, and the cell proliferation rate of dynamic groups were lower than that of the control group. The cell proliferation rate of the group with the frequency of $1 \mathrm{~Hz}$ (group S) was significantly higher than the other two groups with a frequency of $0.25 \mathrm{~Hz}$ or $0.5 \mathrm{~Hz}$ (group SF1 or group SF2).

At the end of the second and fourth weeks, the samples were digested using the method as described in section 2.4.3, and the matrix was extracted and the content of GAGs and the collagen in the matrix was shown in Fig. 6 and Fig. 7. Results showed that the GAGs content in the dynamically cultured groups except the group SA1 and group SA2 were significantly higher than that of the control group. The content of GAGs in the group with a compression strain amplitude of $20 \%$ (group S) was significantly higher than that in the groups with a strain amplitude of $5 \%$ or $10 \%$ (group SA1 or SA2). The collagen content in all dynamic culturing groups were significantly higher than that in the control group, but there was no significant difference in the collagen content between individual dynamically culturing groups at the 4 weeks. The collagen content in the sliding compression group (group S) was higher than that in the 
simple compression group (group P).

The elastic modulus of the samples at 0,2 and 4 weeks was derived from the stress-strain curve tested using the Mechanical Experiment Machine, as shown in Fig. 8. There is no difference observed in the elastic modulus of each group of samples at the week 0 , so the measured elastic modulus value was taken as the initial elastic modulus. The elastic modulus of the specimens decreased with the culture time for all groups, and there was no significant difference between the various loading groups at the same examination point. The elastic modulus of the samples in dynamically culturing groups were lower than that of the control group.

\section{Discussion}

In this study, a method for preparing a calcium alginate hydrogel with a regular shape via crosslinking sodium of alginate with a calcium chloride solution at room temperature was developed. Young rabbit chondrocytes were isolated and seeded into the alginate gel to prepare the TEC specimen. The results showed that the chondrocytes in the scaffolds were in good condition and maintained a normal morphology, which confirmed the feasibility of the process for preparing TEC samples. A cartilage tissue biosimulator that could apply periodic vertical compression movement with horizontal reciprocating motion on the specimens was constructed, which realized coupling of compression, friction and shearing mechanical stimulation. Moreover, the effects of the loading form(groups C, P, and S), compressive strain amplitude(groups SA1, SA2, SA3, and C) and loading frequency (groups SF1, SF2, SF3, and C) on tissue engineering chondrocyte activity, cell proliferation, matrix secretion and elastic modulus were evaluated. In the tissue viability test, the dead and alive cell staining results at week 0 showed that the chondrocytes in all groups were uniformly distributed and in good condition, which confirmed the feasibility of the tissue-engineered cartilage preparation process used in this study. Although dead cells gradually 
appeared in each group at the second and fourth weeks, the living cells still accounted for the majority, which showed that the samples in each group survived well during the dynamic cultivation process. However, the chondrocytes did not spread in the calcium alginate scaffold and there were no significant differences between the control group (group C) and the experimental groups in the morphology and distribution of the cells.

The compression experiment results showed that there was not a positive correlation between the elastic modulus and the matrix content. The elastic modulus of the samples gradually decreased with time because the mechanical properties of tissue-engineered cartilage are primarily determined by the cellular scaffold and the calcium alginate polymer in the scaffolds was degraded. Another reason for the elastic modulus decrease was the lack of barrier protection between the macromolecules in the matrix and the culture medium, which allowed the matrix secreted by the chondrocytes to easily diffuse into the culture medium. Collagen fibres could not limit proteoglycan absorption of water and the consequent swelling. Therefore, the internal matrix content of the tissue-engineered cartilage increased and the mechanical properties did not improve as expected[31].

Static and dynamic compression loads were applied to the specimens to simulate the knee cartilage under standing and exercising conditions. The cell proliferation test results showed that dynamic mechanical stimulation in the first two weeks promoted the proliferation of seed cells, and the sliding compression was more favourable to the promotion of the cells. From the view of nutritional supply, dynamic compression and sliding compression both stimulated the flow of nutrient solution in the tissueengineered cartilage. Therefore, the nutritional supply of tissue engineering cartilage in the experimental groups (groups P, and S) was better than that in the static control group (group C). The number of cells in each group decreased from week 2 to week 4, which indicated that the number of cells in the tissue- 
engineered cartilage reached a peak value between week 0 and week 4 . At 4 weeks, the number of cells in group $\mathrm{P}$ and group $\mathrm{S}$ was less than that in group $\mathrm{C}$, and the reason might be that the time of at which the peak number of cells was reached under mechanical stimulation was earlier than that in the static control group. Histological results showed that the chondrocytes of group $\mathrm{P}$ and group S were more likely to gather together, similar to what can be found in the natural cartilage tissue. This might indicate that mechanical action causes physical migration of cells, which was friendly with regard to the functionalization of the chondrocytes. The results of matrix content testing showed that dynamic mechanical stimulation promoted the secretion of GAGs and collagen by chondrocytes. By comparing the amount of GAG amount in groups $\mathrm{P}, \mathrm{S}$ and $\mathrm{C}$, it could be concluded that the dynamic compressive stress was the main mechanical factor regulating the secretion of GAGs in the matrix.

The effect of compression stress amplitude on cell proliferation in each group was significant. The primary chondrocytes used in this study cannot proliferate indefinitely and the cell proliferative ability decreased with time. Compared with high-amplitude (group S) dynamic compression, low-amplitude sliding compression (group SA1) could not effectively promote flow of the culture medium in threedimensional cell scaffold during dynamic culture. Therefore, nutrient and metabolite exchange was blocked under the low-amplitude sliding compression situation, which resulted in the lowest cell proliferation rate in group SA1. Similarly, the secretion of GAGs in tissue-engineered cartilage also increased with increasing strain amplitude.

The change in loading frequency also had effects on cell proliferation. In the range of $0.25 \mathrm{~Hz}$ to $1 \mathrm{~Hz}$ (groups SF1, SF2, and S), low frequency sliding compression load was not conducive to cell proliferation. From the perspective of nutrient penetration, the high frequency sliding compression load promoted entry of the culture medium into the scaffold, which was the main cause of the low cell proliferation rate in the 
lower frequency group. Matrix content testing showed that sliding compression loads of different frequencies all promoted the secretion of GAGs, and there was no significant difference in the promotion of GAG secretion. However, the change in loading frequency did not affect collagen secretion until the fourth week.

Although this study quantified the effect of dynamic compressive load and shear stress on the secretion and elastic modulus of tissue-engineered cartilage from the perspective of the stress mode of the cartilage, it was still insufficient. The selection of calcium alginate as the scaffold material cannot avoid degradation of the scaffold material and the loss of the matrix secreted by the cartilage when the samples were cultured in vitro, which caused the mechanical properties of the calcium alginate scaffolds to decrease with time. In addition, the biosimulator built in this study cannot measure or provide feedback on the friction of the tissue in real time, nor could it apply a rolling compression load to the samples. These issues need to be improved in future studies.

\section{Conclusion}

The secretion of extracellular matrix in tissue-engineered cartilage is essential for regeneration and functional reconstruction of articular cartilage defects. A bespoke biosimulator was built to incorporate the coupled motion of compression, friction and shear. The results indicated that, in the range of 5\% $20 \%$ of the amplitude of strain and a frequency range of $0.25 \sim 1 \mathrm{~Hz}$, a larger strain and higher frequency were more favourable for the specimen in terms of the tissue bioactivities and extracellular matrix synthesis. Moreover, the combined mechanical stimulation was more beneficial to matrix secretion than the single loading motion. However, the contribution of the combined mechanical stimulation to the engineered cartilaginous tissue matrix was not sufficient to impede the biodegradation of the tissue with culture time. 


\section{References}

1. Sophia Fox AJ, Bedi A, Rodeo SA (2009) The basic science of articular cartilage: structure, composition, and function. Sports health $1(6): 461-468$

2. Hayes DW, Jr., Brower RL, John KJ (2001) Articular cartilage. Anatomy, injury, and repair. Clinics in podiatric medicine and surgery $18(1): 35-53$

3. Musumeci G, Loreto C, Castorina S, Imbesi R, Castrogiovanni P (2013) Current concepts in the treatment of cartilage damage. A review. $118(2): 189-203$

4. Murawski CD, Kennedy JG (2013) Operative Treatment of Osteochondral Lesions of the Talus. Journal of Bone and Joint Surgery-American Volume 95A (11):1045-1054

5. Lan W, Chen W, Huang D (2019) Research progress on osteochondral tissue engineering. Journal of biomedical engineering 36 (3):504-510

6. Sahni V, Tibrewal S, Bissell L, Khan WS (2015) The Role of Tissue Engineering in Achilles Tendon Repair: A Review. Current Stem Cell Research \& Therapy 10 (1):31-36

7. Wang YJ, Shang SH, Li CZ (2016) Aligned Biomimetic Scaffolds as a New Tendency in Tissue Engineering. Current Stem Cell Research \& Therapy 11 (1):3-18

8. Xu Y, Guo X, Yang S, Li L, Mi S (2018) Construction of bionic tissue engineering cartilage scaffold based on 3D printing and oriented frozen technology. Journal of Biomedical Materials Research Part A 106 (6):1664-1676

9. Temenoff JS, Mikos AG (2000) Review: tissue engineering for regeneration of articular cartilage. Biomaterials 21 (5):431-440

10. Wang YZ, Kim UJ, Blasioli DJ, Kim HJ, Kaplan DL (2005) In vitro cartilage tissue engineering with 3D porous aqueous-derived silk scaffolds and mesenchymal stem cells. Biomaterials 26 (34):7082-7094

11. Raghunath J, Salacinski HJ, Sales KM, Butler PE, Seifalian AM (2005) Advancing cartilage tissue engineering: the application of stem cell technology. Current Opinion in Biotechnology 16 (5):503-509

12. Tan AR, Hung CT (2017) Concise Review: Mesenchymal Stem Cells for Functional Cartilage Tissue Engineering: Taking Cues from Chondrocyte-Based Constructs. Stem Cells Translational Medicine 6 (4):1295-1303

13. Niu HJ, Wang Q, Wang YX, Li A, Sun LW, Yan Y, Fan F, Li DY, Fan YB (2012) The study on the mechanical characteristics of articular cartilage in simulated microgravity. Acta Mechanica Sinica 28 (5):1488-1493 14. Chowdhury TT, Bader DL, Shelton JC, Lee DA (2003) Temporal regulation of chondrocyte metabolism in agarose constructs subjected to dynamic compression. Archives of Biochemistry and Biophysics 417 (1):105-111

15. Ikenoue T, Trindade MCD, Lee MS, Lin EY, Schurman DJ, Goodman SB, Smith RL (2003) Meehanoregulation of human articular chondrocyte aggrecan and type II collagen expression by intermittent hydrostatic pressure in vitro. Journal of Orthopaedic Research 21 (1):110-116

16. De Croos JNA, Dhaliwal SS, Grynpas MD, Pilliar RM, Kandel RA (2006) Cyclic compressive mechanical stimulation induces sequential catabolic and anabolic gene changes in chondrocytes resulting in increased extracellular matrix accumulation. Matrix Biology 25 (6):323-331

17. Wu JZ, Herzog W (2006) Analysis of the mechanical behavior of chondrocytes in unconfined compression tests for cyclic loading. Journal of Biomechanics 39 (4):603-616

18. Wagner DR, Lindsey DP, Li KW, Tummala P, Chandran SE, Smith RL, Longaker MT, Carter DR, Beaupre GS (2008) Hydrostatic pressure enhances chondrogenic differentiation of human bone marrow stromal cells in osteochondrogenic medium. Annals of Biomedical Engineering 36 (5):813-820 
19. Omata S, Sonokawa S, Sawae Y, Murakami T (2012) Effects of both vitamin C and mechanical stimulation on improving the mechanical characteristics of regenerated cartilage. Biochemical and Biophysical Research Communications 424 (4):724-729

20. Shelton JC, Bader DL, Lee DA (2003) Mechanical conditioning influences the metabolic response of cell-seeded constructs. Cells Tissues Organs 175 (3):140-150

21. Nebelung S, Gavenis K, Rath B, Tingart M, Ladenburger A, Stoffel M, Zhou B, Mueller-Rath R (2011) Continuous cyclic compressive loading modulates biological and mechanical properties of collagen hydrogels seeded with human chondrocytes. Biorheology 48 (5-6):247-261

22. Chen T, Buckley M, Cohen I, Bonassar L, Awad HA (2012) Insights into interstitial flow, shear stress, and mass transport effects on ECM heterogeneity in bioreactor-cultivated engineered cartilage hydrogels. Biomechanics and Modeling in Mechanobiology 11 (5):689-702

23. Natenstedt J, Kok AC, Dankelman J, Tuijthof GJ (2015) What quantitative mechanical loading stimulates in vitro cultivation best? Journal of experimental orthopaedics 2 (1):15

24. Chen C, Tambe DT, Deng LH, Yang L (2013) Biomechanical properties and mechanobiology of the articular chondrocyte. American Journal of Physiology-Cell Physiology 305 (12):C1202-C1208

25. Wang L, Shen H, Nie JC, Li DC, Fan HB, Jin ZM, Liu CZ (2018) Functional testing on engineered cartilage to identify the role played by shearing. Med Eng Phys 51:17-23

26. Wang L, Li D, He J, Lu B (2018) Research center of biomanufacturing in Xi'an Jiaotong University. BioDesign and Manufacturing 1 (4):280-288

27. Grandolfo M, D'Andrea P, Paoletti S, Martina M, Silvestrini G, Bonucci E, Vittur F (1993) Culture and differentiation of chondrocytes entrapped in alginate gels. Calcified Tissue International 52 (1):42-48

28. Fragonas E, Valente M, Pozzi-Mucelli M, Toffanin R, Rizzo R, Silvestri F, Vittur F (2000) Articular cartilage repair in rabbits by using suspensions of allogenic chondrocytes in alginate. Biomaterials 21

(8):795-801

29. Wang S, An W, Yao Y, Chen R, Zheng X, Yang W, Zhao Y, Hu X, Jiang E, Bie YJJoC (2015) Interleukin 37 Expression Inhibits STAT3 to Suppress the Proliferation and Invasion of Human Cervical Cancer Cells. 6 (10):962-969

30. Ling Wang HS, Jichang Nie, Dichen Li, Hongbin Fan, Zhongmin Jin, Chaozong Liu (2018) Functional testing on engineered cartilage to identify the role played by shearing. Medical Engineering Physics 51:17-23

31. Sittinger M, Lukanoff B, Burmester GR, Dautzenberg H (1996) Encapsulation of artificial tissues in polyelectrolyte complexes: Preliminary studies. Biomaterials 17 (10):1049-1051 
Table 1 Experimental groupings and loading parameter settings for biomimetic mechanical stimulation

\begin{tabular}{cccc}
\hline Groups & $\begin{array}{c}\text { Compressive Strain } \\
\text { Amplitude }\end{array}$ & $\begin{array}{c}\text { Frictional } \\
\text { Velocity } / \mathrm{mm} \cdot \mathrm{s}^{-1}\end{array}$ & $\begin{array}{c}\text { Loading } \\
\text { Frequency } / \mathrm{Hz}\end{array}$ \\
\hline P & $20 \%$ & 0 & 1 \\
S & $20 \%$ & 10 & 1 \\
SF2 & $20 \%$ & 5 & 0.5 \\
SF1 & $20 \%$ & 2.5 & 0.25 \\
SA2 & $10 \%$ & 10 & 1 \\
SA1 & $5 \%$ & 10 & 1 \\
C & 0 & 0 & 0 \\
\hline
\end{tabular}



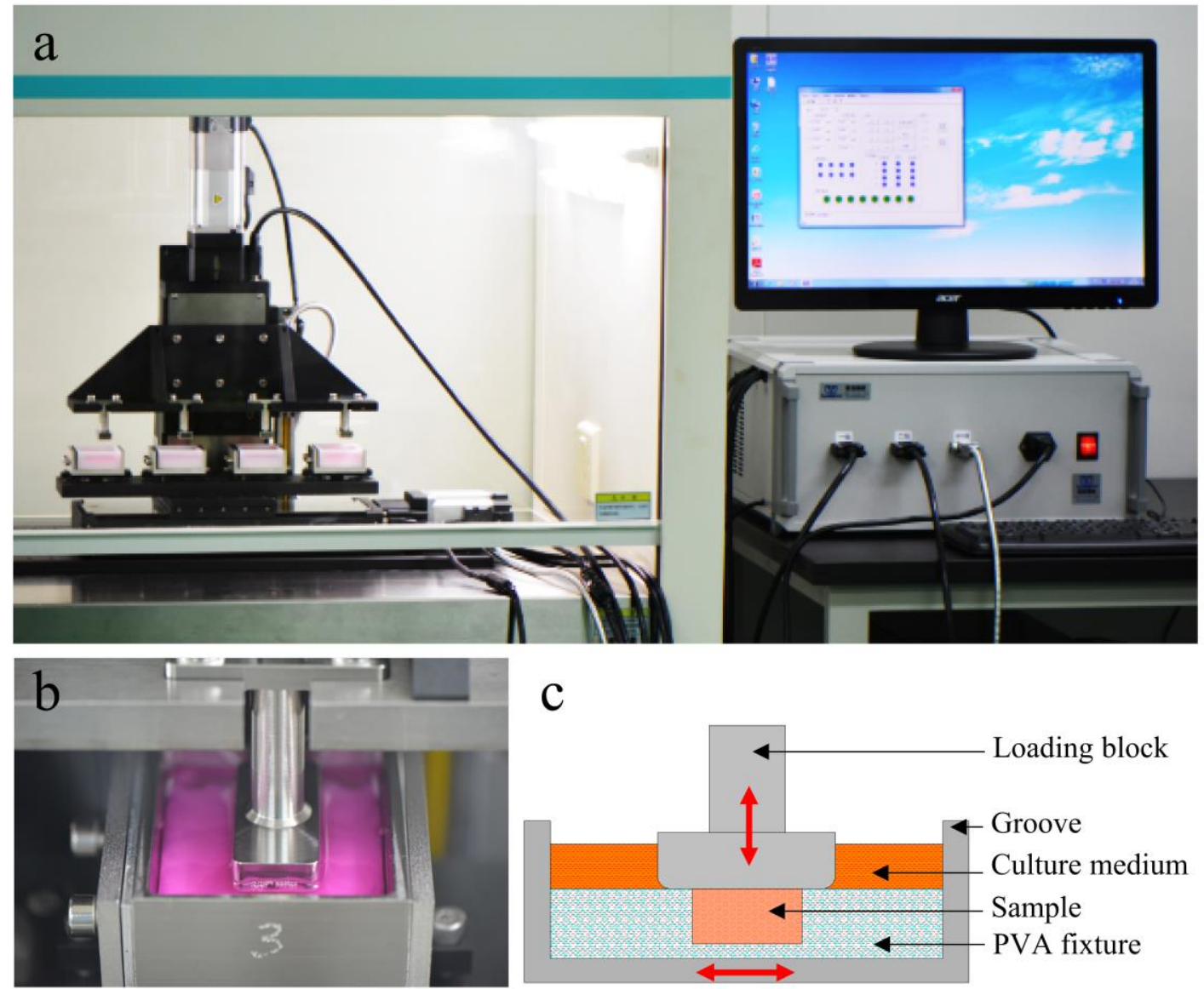

$\mathrm{C}$

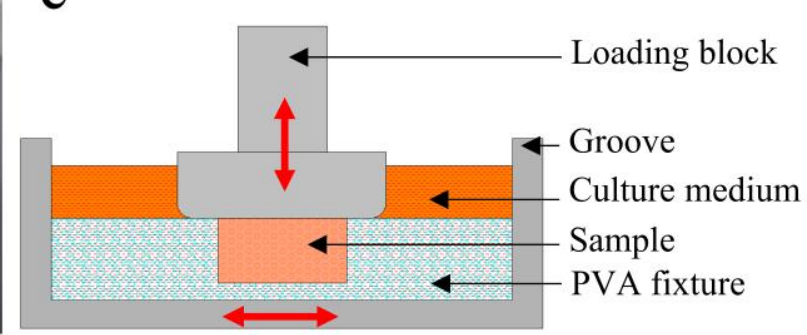

Fig.1. Tissue-engineered cartilage biosimulator (a. Bespoke tissue-engineered cartilage biosimulator; b. Station and fixture of the biosimulator; c. Schematic drawing of the loading scheme) 

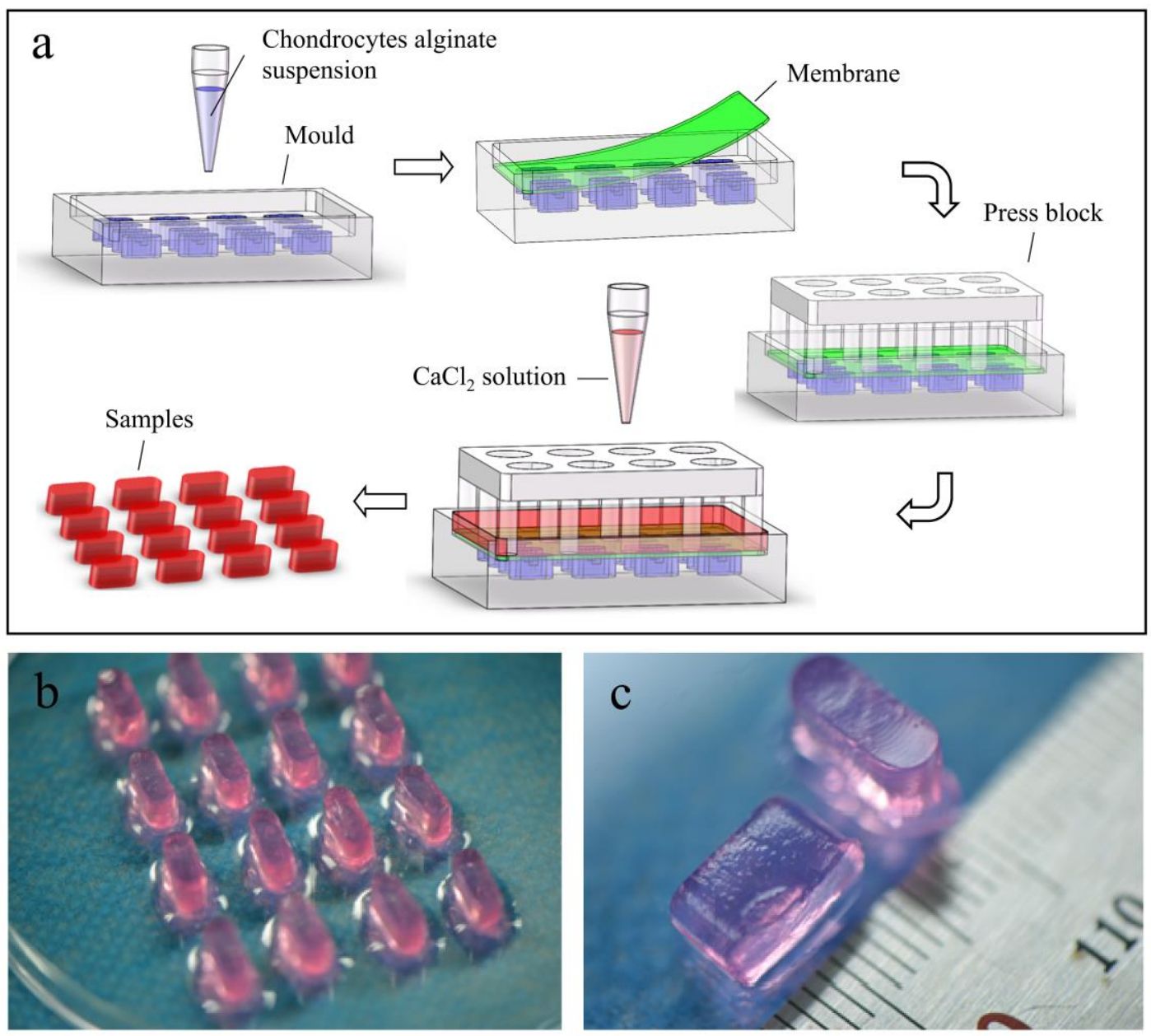

Fig.2. Preparation of tissue-engineered cartilage (a. Preparation of tissue-engineered cartilage;b/c. Samples of the tissue-engineered cartilage) 
0 weeks

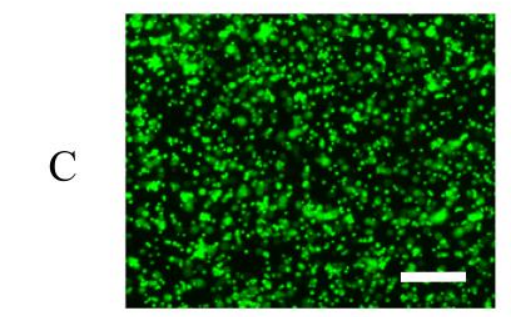

P

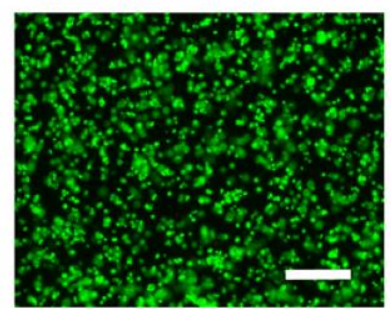

S
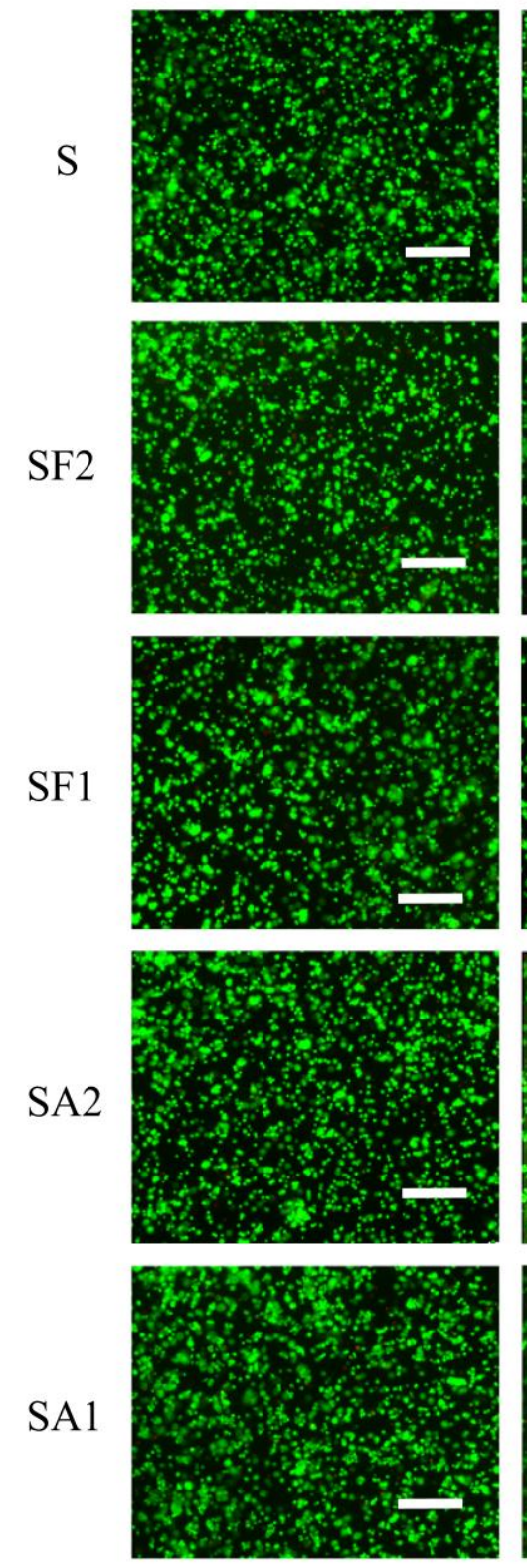

2 weeks
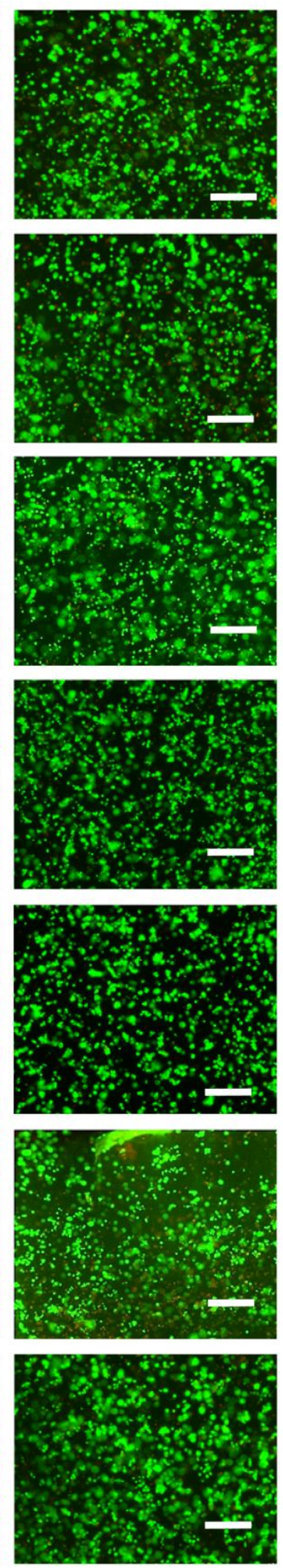

4 weeks
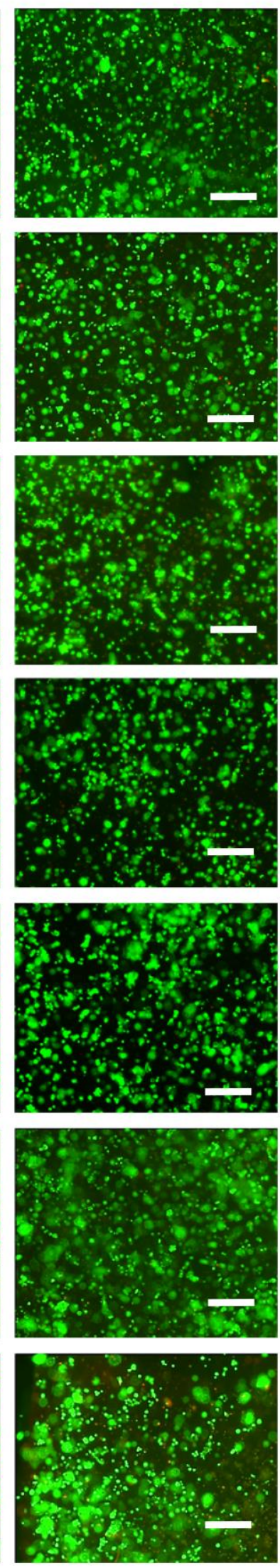

Fig.3. Live/Dead staining results for samples from different groups at 0, 2, and 4 weeks of culture time. Scale bar: $300 \mu \mathrm{m}$. 


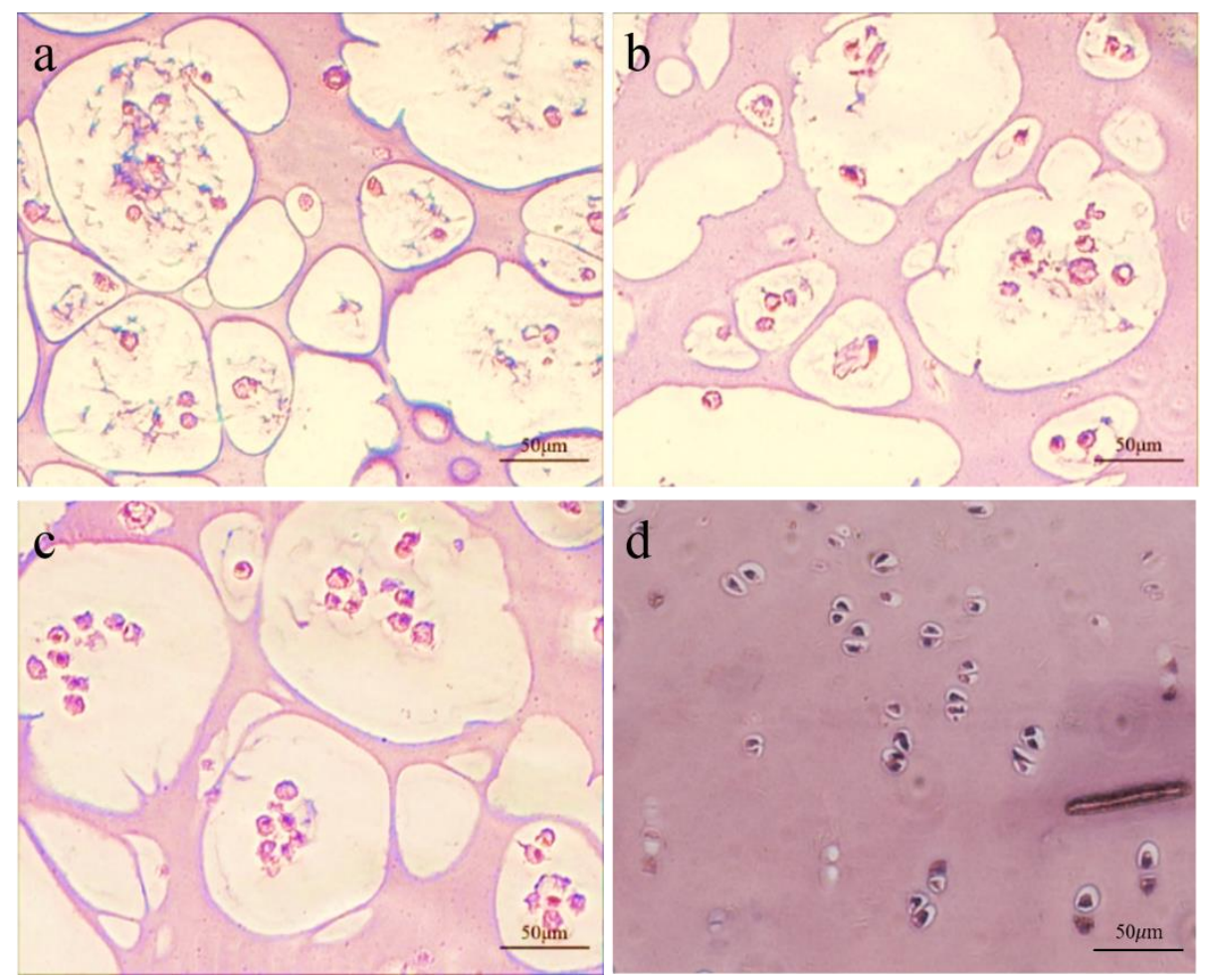

Fig.4. H\&E staining of tissue-engineered cartilage in (a) C, (b) P, and (c) S groups, and (d) natural articular cartilage 


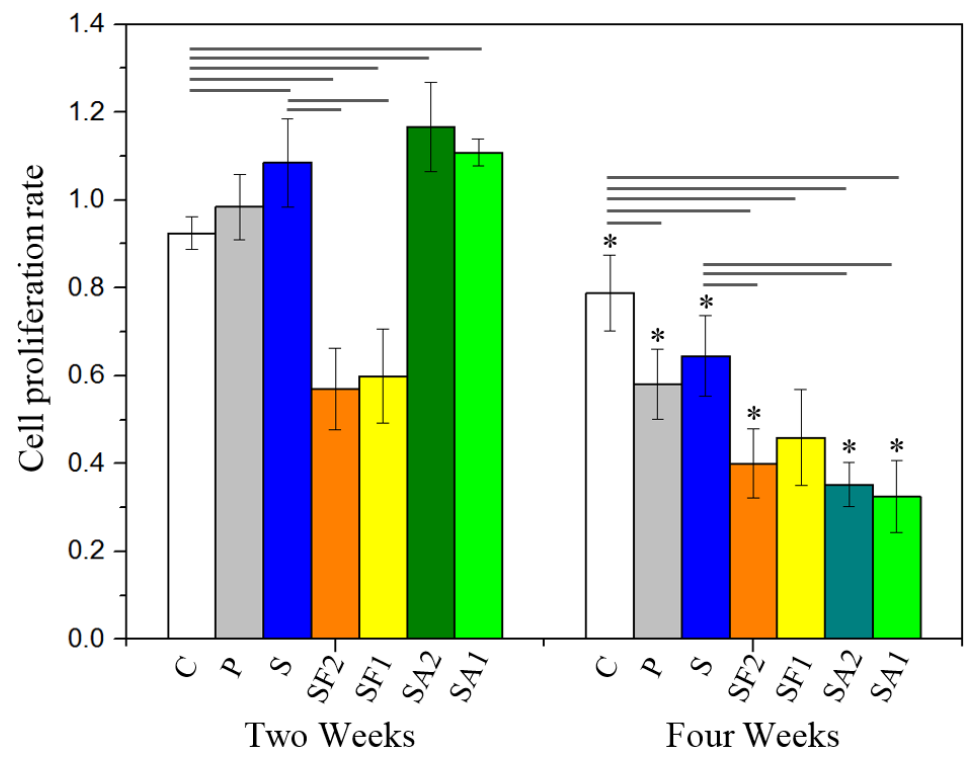

Fig.5. Results of cell proliferation test ("- " indicates that there is significant difference between the detection results of different stimuli groups at the same time point, one-way ANOVA, $\mathrm{p}<0.05, \mathrm{n}=4$; “ * " indicates that the detection result of the same stimuli group is significantly different between the 4 th week and the 2nd week, one-way ANOVA, $\mathrm{p}<0.05, \mathrm{n}=4$ ) 

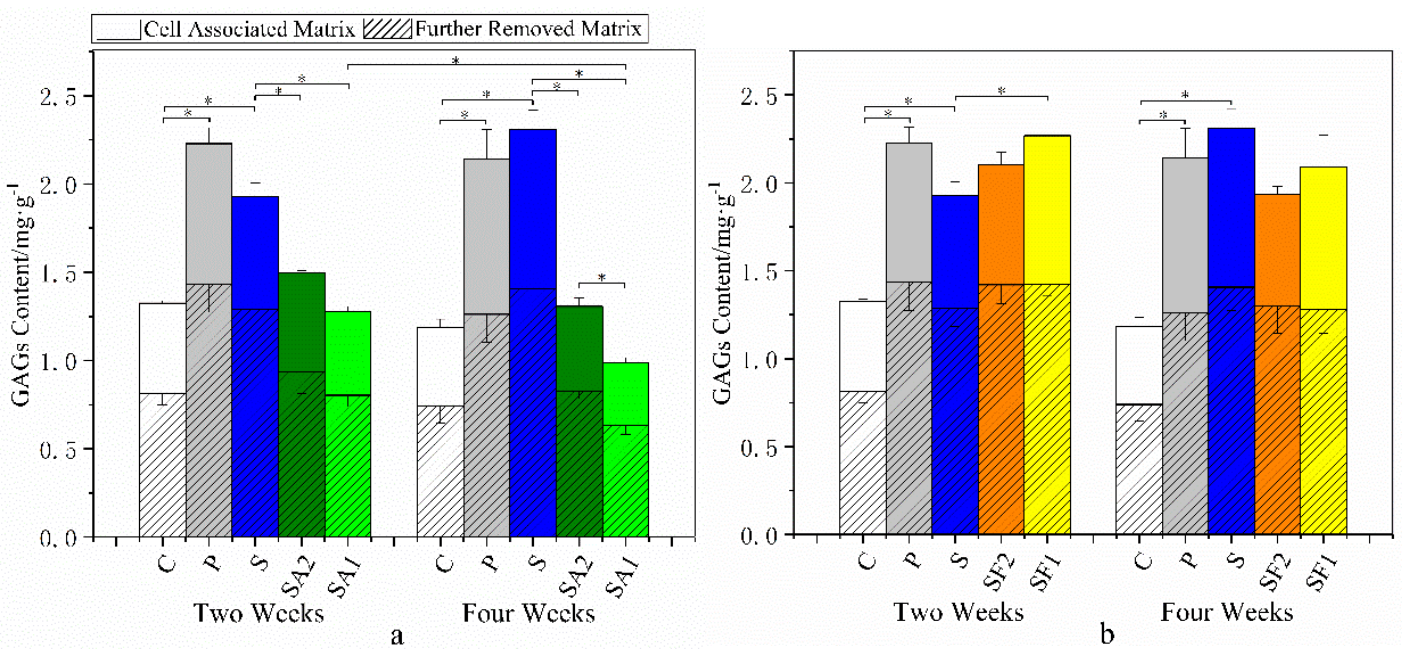

Fig.6. Comparisons of GAGs content in different groups (a. Different compression amplitude groups; b. Different frequency groups) (*: one-way ANOVA, $\mathrm{p}<0.05, \mathrm{n}=4)$ 


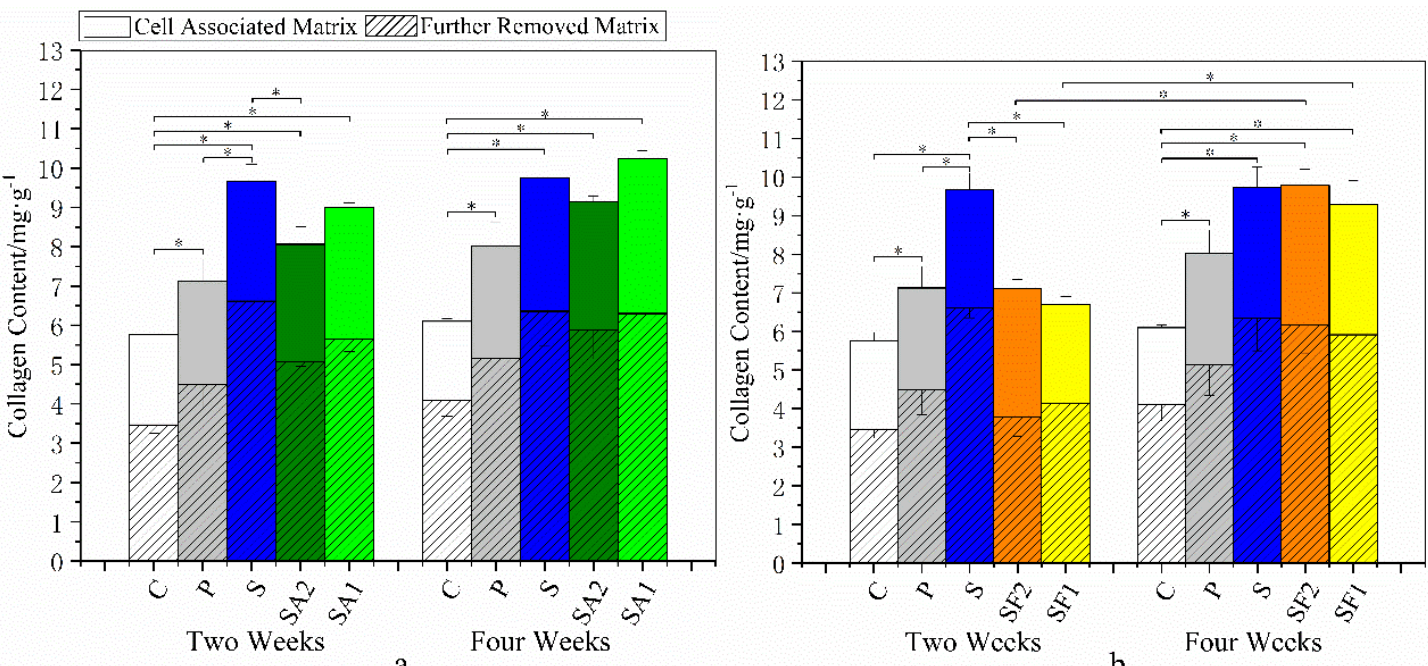

Fig.7. Comparisons of collagen content in different groups (a. Different compression amplitude groups; b. Different frequency groups) (*: one-way ANOVA, $\mathrm{p}<0.05, \mathrm{n}=4$ ) 

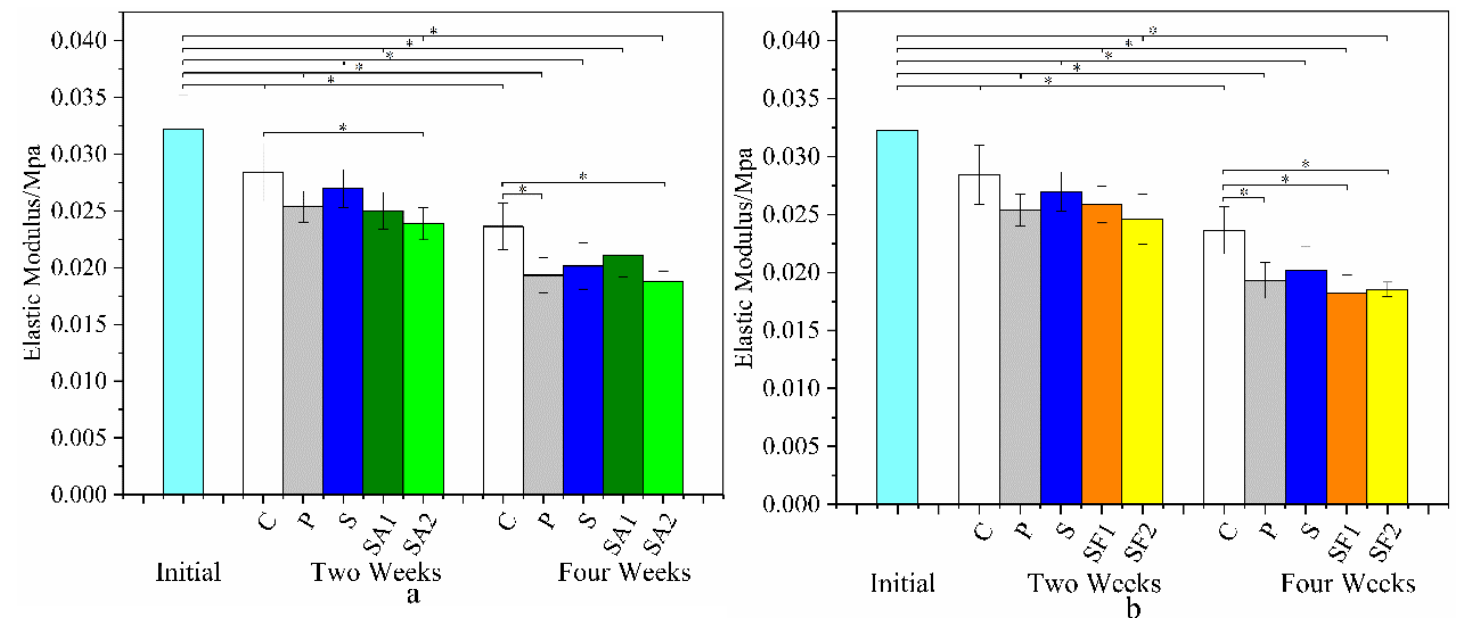

Fig.8. Comparisons of elastic modulus in different groups (a. Different compression amplitude groups; b. Different frequency groups) (*: one-way ANOVA, $\mathrm{p}<0.05, \mathrm{n}=4$ ) 
Click here to access/download Supplementary Material Authorship form_imprints various- pre-acc.pdf 
Click here to access/download Supplementary Material cover letter.docx 
Click here to access/download Supplementary Material Response to Reviewers.docx 
Click here to access/download

Supplementary Material title page.docx 